Pacific Journal of Mathematics

ON THE ENDOMORPHISM RING OF AN ABELIAN $p$-GROUP, 


\title{
ON THE ENDOMORPHISM RING OF AN ABELIAN $p$-GROUP, AND OF A LARGE SUBGROUP
}

\author{
G. S. MoNK
}

\begin{abstract}
For an abelian $p$-group $G$, denote the endomorphism ring of $G$ by $E(G)$, the ideal of small endomorphisms by $E_{s}(G)$ and the quotient ring $E(G) / E_{s}(G)$ by $S(G)$. It is not difficult to show that for a large subgroup $L$ of $G$, the map that sends an endomorphism of $G$ to its restriction on $L$ induces a monomorphism $S(G) \rightarrow S(L)$. We show that if $B_{1}$ is a large subgroup of a group $B_{2}$ which is a direct sum of cyclic $p$-groups and is of cardinality not more than $2 \aleph_{0}$ and $R_{1}$ and $R_{2}$ are suitable subgroups of $E\left(B_{1}\right)$ and $E\left(B_{2}\right)$, then there are groups $G_{1}$ and $G_{2}$ having $B_{1}$ and $B_{2}$ as basic subgroups such that $G_{1}$ is large in $G_{2}$ and $S\left(G_{i}\right) \cong R_{\imath} /\left(E_{s}\left(B_{i}\right) \cap R_{\imath}\right)$, $(i=1,2)$.
\end{abstract}

As with Corner's result [2, Th.2.1], much of the value of this theorem is in producing examples. Although it is clear that these theorems can be used to exhibit counterexamples to several obvious conjectures, we will confine ourselves to giving a counterexample to a theorem of Paul Hill which asserts that the endomorphism ring of a $p$-group $G$ (with $p \neq 2$ ) is generated by its units if this property holds for any large subgroup of $G$. To show that our example has the necessary properties we give, in $\S 3$, some conditions equivalent to a ring of endomorphisms being generated by its units.

All groups considered will be abelian. For a $p$-group $G$, we will denote the set of automorphisms of $G$ by $A(G)$ and the subring of $E(G)$ that $A(G)$ generates by $\{A(G)\}$. The ideal of endomorphisms that annihilate the socle of $G$ will be denoted by $N(G)$. As we will be writing morphisms on the right, the restriction of an endomorphism $\alpha$ of a group $G$ to a fully invariant subgroup $L$ of $G$ will be written as $(L \mid \alpha)$. For information on large subgroups and small homomorphisms the reader is referred to Pierce's paper [6]. The topology on a group or its endomorphism ring will always be the $p$-adic topology. The closure of a set $X$ will be denoted by $X^{-}$.

2. An extension of Corner's Theorem. Given a torsion complete $p$-group $C$ with unbounded basic subgroup $B$ of cardinality not more than $2^{\aleph_{0}}$, Corner's theorem [2, Th. 2.1] gives sufficient conditions on a subring $R$ of $E(B)$ for there to exist a pure subgroup $G$ of $C$ containing $B$ such that $E(G)=E_{s}(G) \oplus R$. It is necessary for our example in section 4 to weaken slightly the conditions on the ring $R$. In doing this we obtain a group $G$ such that $E(G)=E_{s}(G)+R$ without neces- 
sarily having $E_{s}(G) \cap R=(0)$, so that $S(G) \cong R /\left(E_{s}(B) \cap R\right)$. But as will be seen in the example, there is no real loss in this. After we have given the necessary extension of Corner's theorem, we will show that if $B$ is a large subgroup of a basic subgroup $B^{\prime}$ of a torsion complete group $C^{\prime}$ (containing $C$ ) and $R$ is a suitable ring of extensions of the elements of $R$ to $B^{\prime}$, then there is a pure subgroup $G^{\prime}$ of $C^{\prime}$ containing $B^{\prime}$, having $G$ as a large subgroup and such that $E\left(G^{\prime}\right)=$ $E_{s}\left(G^{\prime}\right)+R^{\prime}$.

Definition. If $\alpha$ and $\beta$ are endomorphisms of a group $G$ and $\left(p^{n} G\right)[p](\alpha-\beta)=0$ for some integer $n$, then we will say that $\alpha$ is eventually equal to $\beta$ on $G[p]$ and write $\alpha \doteq \beta$.

We begin with one extension of Corner's theorem that is seen to hold by simply looking at his proof, but is not quite the theorem we need.

Lemma 1. Let $C$ be a torsion complete p-group with an unbounded basic subgroup $B$ of cardinality not more than $2^{\aleph_{0}}$ and let $\Phi$ be a closed separable subgroup of $E(B)$ such that $1 \in \Phi$ and

$\left(C_{1}\right)$. If $\varphi_{1}, \varphi_{2} \in \Phi$, there is an element $\varphi_{3} \in \Phi$ and a small endomorphism $\sigma \in E_{s}(B)$ such that $\varphi_{1} \varphi_{2}=\varphi_{3}+\sigma$.

$\left(C_{2}\right)$. If $\varphi \in \Phi$ and $\varphi \doteq 0$, then $\varphi \in p \Phi$.

Then there is a pure subgroup $G$ of $C$ containing $B$ such that $E(G)=$ $E_{s}(G) \oplus \Phi$.

Proof. This is just Corner's theorem with the assumption that $\Phi$ be closed under multiplication replaced by $\left(C_{1}\right)$. But the only place in which the multiplicative closure of $\Phi$ is used is in the proof of his Lemma 2.10 in which he has a subset $X_{r}$ of $C$ and a $\Phi$-invariant subgroup $H$ of $C$ containing $B$ and he wants to conclude that $H_{r}=H+$ $\Sigma\left\{x_{i} \Phi \mid x_{i} \in X_{r}\right\}$ is a $\Phi$-invariant subgroup of $C$. That it is a subgroup is a consequence of the fact that $\Phi$ is a group. On the other hand, for $h$ in $H_{r}$, there is an element $h^{\prime}$ in $H$ and a finite set of elements $\left\{\varphi_{i}\right\}_{0}^{m}$ of $\Phi$ such that $h=h^{\prime}+\sum_{0}^{m} x_{i} \varphi_{i}$, whence, for any $\varphi \in \Phi,(h) \varphi=h^{\prime} \varphi+$ $\sum_{0}^{m} x_{i}\left(\varphi_{i} \varphi\right)$. Now by condition $\left(C_{1}\right)$, there is, for each $i$, an element $\varphi_{i}^{\prime} \in \Phi$ and a small endomorphism $\sigma_{i}$ of $B$ such that $\varphi_{i} \varphi=\varphi_{i}^{\prime}+\sigma_{i}$. However, since $\sigma_{i} \in E_{s}(B)$, we know, from [6, Th. 4.4] that $(C) \sigma_{i} \subseteq B$, from which we conclude that $\left(x_{i}\right) \sigma \in B \cong H$ and

$$
(x) \varphi=\left(h^{\prime}\right) \varphi+\sum_{1}^{m}\left(x_{i}\right) \sigma_{i}+\sum_{1}^{m}\left(x_{i}\right) \varphi_{i}^{\prime} \in H+\sum\left\{x_{i} \Phi \mid x_{i} \in X_{r}\right\} .
$$

LEMMA 2. Given any p-group $G, E_{s}(G)$ is complete, and there is a torsion free, complete subgroup $K$ of $E(G)$ such that $E(G)=E_{s}(G) \oplus K$ and $1 \in K$.

Proof. That $E_{s}(G)$ is pure and complete in $E(G)$ and that $E(G) / E_{s}(G)$ 
is torsion free are shown by Pierce [6, Th. 7.5]. It is then easily seen that $E_{s}(G) \oplus R_{p} \cdot 1$ is pure and torsion complete in $E(G)$ (where $R_{p}$ is the $p$-adic integers) so that it has a complement $K^{\prime}$ in $E(G)$. The group $K=R_{p} \cdot 1 \oplus K^{\prime}$ is then the desired complement of $E_{s}(G)$.

THEOREM 1. Let $C$ be a torsion complete p-group with an unbounded basic subgroup $B$ of cardinality not more than $2^{\aleph_{0}}$ and let $R_{0}$ be a countable unital subring of $E(B)$ such that

$\left(C^{\prime}\right)$. If $\alpha \in R_{0}$ is such that $\alpha \doteq 0$, there is an element $\beta \in R_{0}$ and a small endomorphism $\sigma$ of $B$ such that $\alpha=p \beta+\sigma$.

Then there is a pure subgroup $G$ of $C$ containing $B$ such that $E(G)=E_{s}(G)+\left(E_{s}(B)+R_{0}\right)^{-}$.

Proof. Using lemma 2, write $E(B)=E_{s}(B) \oplus K$ with $1 \in K$, let $\Phi_{0}=\left(R_{0}+E_{s}(B)\right) \cap K$ and $\Phi=\Phi_{0}^{-}$. Certainly $\Phi$ is a closed separable subgroup of $E(B)$ containing 1 . By the modular law, it is seen that

$$
\Phi_{0}+E_{s}(B)=R_{0}+E_{s}(B) \text {. }
$$

It is evident that since $R_{0}+E_{s}(B)$ is a ring, $\Phi_{0}$ has the property $\left(C_{1}\right)$ of lemma 1 from which it is easily shown that $\Phi$ does also. To show that $\Phi_{0}$ has the condition $\left(C_{2}\right)$ of Lemma 1, suppose that $\phi \in \Phi_{0}$ and $\varphi \doteq 0$. By (1) we can choose $\psi \in R_{0}$ and $\sigma \in E_{s}(B)$ such that $\varphi=$ $\psi+\sigma$, so that $\psi \doteq 0$ and by $\left(C^{\prime}\right), \psi=p \psi^{\prime}+\sigma^{\prime}$ for some $\psi^{\prime} \in R_{0}$ and $\sigma^{\prime} \in E_{s}(B)$. Applying (1) again then gives $\varphi=p \varphi^{\prime}+\sigma^{\prime \prime}$ with $\varphi^{\prime} \in \Phi_{0}$ and $\sigma^{\prime \prime} \in E_{s}(B)$. Inasmuch as $\Phi_{0} \cap E_{s}(B)=(0)$, we infer that $\varphi=p \varphi^{\prime} \in$ $p \Phi_{0}$. Now, if $\varphi \in \Phi$ and $\varphi \doteq 0$ there is a sequence $\left\{\varphi_{i}\right\}_{1}^{\infty}$ of elements of $\Phi_{0}$ that converges to $\varphi$. The endomorphisms $\varphi_{i}$ can be chosen such that $\varphi-\varphi_{i} \in p K$ for every $i$, so that $\varphi_{i} \doteq 0$ and there is an element $\varphi_{i}^{\prime} \in R_{0}$ such that $\varphi_{i}=p \varphi_{i}^{\prime}$. Using the fact that $K$ is torsion free, it is easily seen that $\left\{\varphi_{i}^{\prime}\right\}_{i=1}^{\infty}$ is a Cauchy sequence which, since $\Phi$ is complete, has a limit $\phi^{\prime} \in \Phi$. Therefore $\varphi=p \phi^{\prime} \in p \Phi$, $\Phi$ has the property $\left(C_{2}\right)$ of Lemma 1 , and we can apply this lemma to obtain a pure subgroup $G$ of $C$ containing $B$ such that $E(B)=E_{s}(G) \oplus \Phi$. Now observe that since $\left(E_{s}(B)+R_{0}\right)^{-}$contains $\Phi_{0}$ and is closed, we have $\left(E_{s}(B)+\right.$ $\left.R_{0}\right)^{-} \supseteqq \Phi$ whence $E(G) \subseteq E_{s}(G)+\left(E_{s}(B)+R_{0}\right)^{-}$. On the other hand $E(G) \supseteqq E_{s}(B)+\Phi_{0}=E_{s}(B)+R_{0}$, so that, since $E(G)$ is closed, $E(G) \supseteqq$ $\left(E_{s}(B)+R_{0}\right)^{-}$, and we conclude that $E(G)=E_{s}(G)+\left(E_{s}(B)+R_{0}\right)^{-}$.

Notation. For an element $x$ in a $p$-group $G$ we will denote the height of $x$ in $G$ by $h_{G}(x)$. The $n^{\text {th }}$ Ulm invariant of $G$ will be denoted by $f_{G}(n)$.

Lemma 3. Let $A$ be a p-group without elements of infinite height 
with subgroups $G, K$ and $L$ such that (i) $G$ is pure in $A$, (ii) $L$ is large in $G$, (iii) $L$ is pure and dense in $K$. Then the subgroup $M=$ $K+G$ of $A$ has $K$ as a large subgroup.

Proof. According to Pierce [6, Th. 2.6], the large subgroups of a group $G$ are exactly the subgroups of the form

$$
G\{n(i)\}=\left\{g \in G \mid h_{G}\left(p^{i} g\right) \geqq n(i), \quad i=0,1,2, \cdots\right\},
$$

for all finite $U$-sequences $\{n(i)\}_{0}^{\infty}$, where a strictly increasing sequence $\{n(i)\}_{0}^{\infty}$ of nonnegative integers is called a finite $U$-sequence for a group $G$ of infinite length if $n(i)+1<n(i+1)$ only if $f_{G}(n(i)) \neq 0$. It is clear that $G$ is pure and dense in $M$ so that the two groups have the same Ulm invariants and every finite $U$-sequence of $G$ is also a finite $U$-sequence of $M$. Thus, if $\{n(i)\}_{0}^{\infty}$ is the fininite $U$-sequence such that

$$
L=\left\{g \in G \mid h_{G}\left(p^{i} g\right) \geqq n(i), \quad i=0,1,2, \cdots\right\},
$$

the lemma will be proved if we can show that

$$
K=\left\{g \in M \mid h_{M}\left(p^{i} g\right) \geqq n(i), \quad i=0,1,2, \cdots\right\} .
$$

First observe that for every $i, n(i) \geqq i$, so by the purity and denseness of $L$ in $K$

$$
K\left[p^{r}\right]=\left(p^{n(i)-i} K\right)\left[p^{r}\right]+L\left[p^{r}\right], \quad r=1,2, \cdots,
$$

Now suppose that $g \in K$ and $i$ is a nonnegative integer. Then by (4) $g=p^{n(i)-i} g_{i}+x_{i}$ with $g_{i} \in K$ and $x_{i} \in L$, so that $p^{i} g=p^{n(i)} g_{i}+p^{i} x_{i}$. But since $x_{i} \in L, p^{i} x_{i}=p^{n(i)} y_{i}$ for some $y_{i} \in G$, whence $h_{M}\left(p^{i} g\right) \geqq n(i)$, and we conclude that $g \in M\{n(i)\}$. Conversely, suppose $z \in M\{n(i)\}$ and $z=x+g$ with $x \in K$ and $g \in G$. Then let $r$ be an integer such that $z, x, g \in A\left[p^{r}\right]$ and choose $x^{\prime} \in K$ and $y \in L\left[p^{r}\right]$ with $x=p^{n(r)-r} x^{\prime}+y$, so that $p^{i}(g+y)=p^{i} z-p^{n(r)-r+i} x^{\prime}$. For $i<r, n(r)-r+i>n(i)$, so that $h_{G}\left(p^{i}(g-y)\right)=h_{M}\left(p^{i}(g-y)\right) \geqq n(i)$, while, for $i \geqq r p^{i}(g-y)=0$, whence $h_{M}\left(p^{i}(g-y)\right) \geqq n(i)$. Thus $g-y \in G|n(i)|=L$ and $g \in L$ from which we conclude that $z \in K$, as was to be shown.

Lemma 4. Let $K$ be a large subgroup of a direct sum $B$ of cyclic p-groups. Then an endomorphism $\alpha$ of $K$ can be extended to an endomorphism $\alpha^{\prime}$ of $B$ if and only if $h_{B}(x) \leqq h_{B}(\alpha(x))$ for every $x \in K$.

Proof. If $\alpha$ has an extension, it clearly does not diminish $B$-height on $K$. By a lemma of Pierce [6, Lemma 2.12], if $B=\oplus \sum_{i \in I}\left(b_{i}\right)$, then $K=\bigoplus \sum_{i \in I}\left(\left(b_{i}\right) \cap K\right)$, so that there is a function $m$ of $I$ into the natural numbers such that $K=\bigoplus \sum_{i \in I}\left(p^{m(i)} b_{i}\right)$. Thus if $\alpha$ does not diminish $B$-height on $K$, given $i \in I$, there is an $x_{i} \in B$ such that 
$\alpha\left(p^{m(i)} b_{i}\right)=p^{m(i)} x_{i}$ and the endomorphism of $B$ given by $\alpha^{\prime}\left(b_{i}\right)=x_{i}$ is the desired extension of $\alpha$.

The following theorem, when taken with Theorem 1 provides the representation of two rings that we seek.

Theorem 2. Suppose $G$ is a p-group with basic subgroup $B$ and $R$ is a subring of $E(B)$ containing $E_{s}(B)$ such that $E(G)=E_{s}(G)+R$ and suppose that $B$ is a large subgroup of the basic subgroup $B^{\prime}$ of the torsion complete group $C^{\prime}$ containing $C$, the torsion completion of $B$. Then there is a pure subgroup $G^{\prime}$ of $C^{\prime}$ containing $B^{\prime}$ having $G$ as a large subgroup and such that $E\left(G^{\prime}\right)=E_{s}\left(G^{\prime}\right)+R^{\prime}$ where $R^{\prime}=$ $\left\{\alpha \in E\left(B^{\prime}\right) \mid(B \mid \alpha) \in R\right\}$.

Proof. We let $G^{\prime}=G+B$, so that by Lemma $3, G$ is large in $G^{\prime}$. Inasmuch as $G^{\prime} / B^{\prime}$ is isomorphic to a quotient group of $G / B$, it is a divisible group. This, together with the fact that $B^{\prime}$ is pure in $C^{\prime}$ yields that $G^{\prime}$ is pure in $C^{\prime}$. Now if $\alpha^{\prime} \in R^{\prime}$ and $\alpha=\left(B \mid \alpha^{\prime}\right)$, then $\alpha \in R \cong E(G)$, so that $\alpha^{\prime} \in E\left(G^{\prime}\right)$. Thus $E\left(G^{\prime}\right) \supseteqq E_{s}\left(G^{\prime}\right)+R^{\prime}$ and it remains to show the reverse inclusion. Given $\alpha^{\prime} \in E\left(G^{\prime}\right)$, let $\alpha=\left(G \mid \alpha^{\prime}\right)$ and let $\sigma \in E_{s}(B)$ and $\beta \in R$ be such that $\alpha=\sigma+\beta$. If $L=\operatorname{ker} \sigma$ and $B_{2}=L \cap B$, we see from [6, Th. 2.8] that $B_{2}$ is large in $B$ and hence by [6, Th. 2.13] that it is large in $B^{\prime}$. Inasmuch as $\beta=\alpha^{\prime}$ on $B_{2}, \beta$ does not diminish $B^{\prime}$-height in $B_{2}$, whence, by Lemma 4 , there is an element $\beta^{\prime} \in E\left(B^{\prime}\right)$ such that $\beta^{\prime}=\beta$ on $B_{2}$. Let $\beta_{1}=\left(B \mid \beta^{\prime}\right)$. Then since $B_{2}\left(\beta_{1}-\beta\right)=0, \beta_{1}=\beta+\sigma_{1}$ for some $\sigma_{1} \in E_{s}(B) \subseteq R$, and $\beta_{1} \in R$ so that $\beta^{\prime} \in R^{\prime}$. Now $\alpha-\beta_{1}=\sigma-\sigma_{1} \in E_{s}(G)$, and there is a large subgroup $K$ of $G$ and hence of $G^{\prime}$ annihilated by $\alpha-\beta_{1}$. Thus $K\left(\alpha^{\prime}-\beta^{\prime}\right)=K(\alpha-\beta)=0$, and we conclude that $\alpha^{\prime}=\beta^{\prime}+\sigma^{\prime}$ for $\beta^{\prime} \in$ $R^{\prime}$ and $\sigma^{\prime} \in E_{s}\left(G^{\prime}\right)$ as was to be shown.

The following bit of information on the relationship between a group and a large subgroup is helpful in constructing examples.

Lemma 5. Let $G$ be a p-group, $\{k(i)\}_{1}^{\infty}$ an increasing sequence of nonnegative integers and $H$ the large subgroup of $G$ given by $H=$ $\sum_{1}^{\infty}\left(p^{k(i)} G\right)\left[p^{i}\right]$. Then

$$
f_{H}(j)=\sum\left\{f_{G}(k(j+1)+j+i) \mid 0 \leqq i \leqq k(j+2)-k(j+1)\right\} .
$$

Proof. Routine calculations show that for any increasing sequence of nonnegative integers $\{m(i)\}_{1}^{\infty}$

$$
\begin{aligned}
\left(\sum_{1}^{r+1}\left(p^{m(i)} G\right)\left[p^{i}\right]\right)[p] & \subseteq\left(p^{m(r+1)} G\right)\left[p^{r}\right]+\sum_{1}^{r}\left(p^{m(i)} G\right)\left[p^{i}\right] \\
& =\sum_{1}^{r}\left(p^{m(i)} G\right)\left[p^{i}\right]
\end{aligned}
$$


so that by induction on $r$ we have $\left(\sum_{1}^{r}\left(p^{m(i)} G\right)\left[p^{i}\right]\right) \subseteq\left(p^{m(1)} G\right)[p]$, and $\left(\sum_{1}^{\infty}\left(p^{m(i)} G\right)\left[p^{i}\right]\right)[p]=\left(p^{m(1)} G\right)[p]$. It is also easily seen that for integers $i, j$ and $k$ with $\left.i<k, p^{i}\left(p^{j} G\right)\left[p^{k}\right]\right)=\left(p^{i+j} G\right)\left[p^{k-i}\right]$. Therefore given any integer $j, p^{j} H=\sum_{i=j+1}^{\infty}\left(p^{k(i)+j} G\right)\left[p^{i-j}\right]$, and $\left(p^{j} H\right)[p]=\left(p^{k(j+1)+j} G\right)[p]$. Inasmuch as the dimension of the vector space

$$
\left(p^{k(j+1)+j} G\right)[p] /\left(p^{k(j+2)+j+1} G\right)[p]
$$

is the sum of the dimensions of the vector spaces

$$
\left(p^{k(j+1)+j+i} G\right)[p] /\left(p^{k(j+2)+j+i+1} G\right)[p]
$$

for $i=0,1,2, \cdots,(k(j+2)-k(j+1))$, we have the desired result.

\section{Endomorphism rings generated by their units.}

LEMma 6. The following conditions are equivalent for an endomorphism $\alpha$ of a p-group G: (a) There is a bounded projection $\pi$ in $E(G)$ such that $(1-\pi) \alpha \in N(G)$, (b) $\alpha \in E_{s}(G)+N(G)$, (c) $\alpha \doteq 0$.

Proof. Inasmuch as a bounded endomorphism is small and for any projection $\pi, \alpha=\pi \alpha+(1-\pi) \alpha$, we see that (a) implies (b). Since (b) obviously implies (c), it remains to show that (c) implies (a). Suppose $\left(p^{n} G\right)[p] \alpha=0$. It is not difficult to show that for any integer $n$ (cf. the proof of Lemma 1 in [5]) there are subgroups $G_{0}$ and $G_{1}$ of $G$ such that $G=G_{0} \oplus G_{1}, p^{n} G_{0}=0$ and $G_{1}[p]=\left(p^{n} G\right)[p]$. Thus, if we let $\pi$ be the projection on $G_{0}$ along $G_{1}$, then $\pi$ is bounded and $G_{0}(1-\pi)=0$ while $(1-\pi) \alpha=\alpha$ on $G_{1}[p]=\left(p^{n} G\right)[p]$, so that $G_{1}[p](1-\pi) \alpha=0$ and $(1-\pi) \alpha \in N(G)$.

THEOREM 3. Given a p-group $G$ with $p \neq 2$, all of the following rings are generated by units if any one of them is: (a) $E(G)$, (b) $E(G) / E_{s}(G)$, (c) $E(G) /\left(E_{s}(G)+N(G)\right)$.

Proof. Since the property of being generated by units is inherited by the quotient rings of a ring, we need only show that if $E(G) /\left(E_{s}(G)+\right.$ $N(G)$ ) is generated by units, then $E(G)$ is also. We first prove that in any case $E_{s}(G)+N(G) \subseteq\{A(G)\}$. If $\sigma \doteq 0$, then let $\pi$ be a finite projection such that $(1-\pi) \sigma \in N(G)$ and write $\sigma=(1-\pi) \sigma+\pi \sigma(1-\pi)+$ $\pi \sigma \pi$. For any endomorphism $\varphi \in N(G), \sum_{0}^{\infty} \varphi^{i}$ is also an endomorphism of $G$, so that $1-\varphi$ has an inverse and $\varphi \in\{A(G)\}$. Thus $(1-\pi) \sigma \epsilon$ $\{A(G)\}$. On the other hand, $\pi \sigma \pi$ can be viewed as an endomorphism of the bounded group $G \pi$ and as such is the sum of automorphisms of this group ([1, Th. 2.3]). But clearly this expression of $\pi \sigma \pi$ as a sum of automorphisms can be extended to $\pi \sigma \pi$ as an endomorphism $G$, so that $\pi \sigma \pi \in\{A(G)\}$. Finally, since $1-\pi \sigma(1-\pi)$ has $1+\pi \sigma(1-\pi)$ 
as an inverse, $\pi \sigma(1-\pi) \in\{A(G)\}$ and we conclude that $\sigma \in\{A(G)\}$ If $\varphi \in E(G)$ is such that $\varphi+E_{s}(G)+N(G)$ is a unit, there is an integer $n$ and an endomorphism $\psi \in E(G)$ such that $\psi \varphi=1=\varphi \psi$ on $\left(p^{n} G\right)[p]$. By a theorem of Pierce [6, Th. 13.1] we then have that $\left(\left(p^{n} G\right) \mid \varphi\right)$ is an automorphism of $p^{n} G$ so that by a theorem of Fuchs [3, Lemma 1], there is an automorphism $\varphi^{\prime}$ of $G$ such that $\varphi=\varphi^{\prime}$ on $p^{n} G$. Thus, $\varphi=\varphi^{\prime}+\sigma$ for some $\sigma \in E_{s}(G)$. Now if we assume that $E(G) /\left(E_{s}(G)+\right.$ $N(G)$ ) is generated by its units and $\alpha$ is in $E(G)$, then there is a set of endomorphisms $\left\{\alpha_{i}\right\}_{1}^{m}$ of $G$ such that $\alpha_{i}+E_{s}(G)+N(G)$ is a unit for every $i$ and $\alpha \doteq \sum_{1}^{m} \alpha_{i}$. By what we just showed, there is, for each $i$, an automorphism $\alpha_{i}^{\prime}$ of $G$ such that $\alpha_{i} \doteq \alpha_{i}^{\prime}$, whence $\alpha \doteq \sum_{1}^{m} \alpha_{i}^{\prime}$ and $\alpha \in\{A(G)\}$.

4. Counterexample. We give in this section, a counterexample to the theorem of $\mathrm{P}$. Hill [4, cor. 4.6] which says that if $L$ is a large subgroup of the p-group $G(p \neq 2)$, then $E(G)=\{A(G)\}$ if and only if $E(L)=\{A(L)\}$. We fix the following notation: $B^{\prime}=\bigoplus \sum_{0}^{\infty}\left(b_{i}^{\prime}\right)$, $\left(E\left(b_{i}^{\prime}\right)=i+1\right)$ is a standard basic $p$-group $(p \neq 2)$ with torsion completion $C^{\prime}$ and $B=\sum_{1}^{\infty}\left(p^{n(i)} B\right)\left[p^{i}\right]$ is a large subgroup of $B^{\prime}$ where $n(1)=$ $n(2)=0$, and $n(i)=\sum\{j \mid 1 \leqq j \leqq i\}$ for $i \geqq 2$. Let $C$ be the torsion completion of $B$ in $C^{\prime}$.

LEMma 7. The group $B$ can be written as $B=\oplus \sum_{1}^{\infty} B_{i}$ where $B_{i}=\bigoplus \sum_{j=0}^{i-1}\left(c_{i j}\right)$, with $E\left(c_{i j}\right)=i$ and $c_{i j}=p^{r(j)} b_{s(j)}^{\prime}$ for $0 \leqq j<i$, for strictly increasing sequences $\{r(j)\}_{j=0}^{i-1}$ and $\{s(j)\}_{j=0}^{i-1}$.

Proof. We infer from [6, Th. 2.12] that

$$
B=\oplus \sum_{0}^{\infty}\left(\left(b_{i}^{\prime}\right) \cap B\right) \text {. }
$$

On the other hand, according to Lemma 5, every direct decomposition of $B$ into cyclic subgroups involves exactly $i$ summands of order $p^{i}$. Thus, if we let $B_{i}$ be the sum of those summands in (1) of order $p^{i}$, we have that $B_{i}=\bigoplus \sum_{0}^{i-1}\left(p^{r(j)} b_{s(j)}^{\prime}\right)$ for some strictly increasing sequence $\{s(j)\}_{0}^{i-1}$. But for $0 \leqq j<k<i, E\left(b_{s(j)}^{\prime}\right)<E\left(b_{s(k)}^{\prime}\right)$ while

$$
E\left(p^{r(j)} b_{s(\jmath)}^{\prime}\right)=E\left(p^{r(k)} b_{s(k)}^{\prime}\right),
$$

so that $r(j)<r(k)$. If we then let $c_{i j}=p^{r(j)} b_{s(j)}^{\prime}$ we obtain the desired result.

Now we give endomorphisms $\left\{P_{i}\right\}_{-\infty}^{\infty},\left\{E_{i}\right\}_{0}^{\infty},\left\{F_{i}\right\}_{1}^{\infty}$, of $B$ by defining them on the basis of each $B_{m}$. In all that follows, when we are restricting attention to a particular $B_{m}$, we will denote by $i \oplus j$ the addition of the integers $i$ and $j$ modulo $m$. 


$$
\begin{aligned}
& \left(c_{m j}\right) P_{i}=c_{m, j \oplus i} \\
& \left(c_{m j}\right) E_{i}=\left\{\begin{array}{l}
0, j \neq i \\
c_{m j}, j=i
\end{array}\right. \\
& \left(c_{m j}\right) F_{i}=\left\{\begin{array}{l}
0, j \neq m-i \\
c_{m j}, j=m-i .
\end{array}\right.
\end{aligned}
$$

Let $R_{0}$ be the subring of $E(B)$ generated by $\left\{P_{i}\right\}_{-\infty}^{\infty},\left\{E_{i}\right\}_{0}^{\infty},\left\{F_{i}\right\}_{1}^{\infty}$. Note that an element of $R_{0}$ gives an endomorphism of each $B_{m}$ and thus has an $m$ by $m$ matrix representation for each $m$. Since we are writing endomorphisms on the right, the $m$ by $m$ matrix corresponding to an element $\varphi$ of $R_{0}$ is the matrix whose $i$ th row is the sequence of coefficients used to represent $\left(c_{m i}\right) \varphi$ where $0 \leqq i<m$. This means that in an $m$ by $m$ matrix we are numbering the rows and columns from 0 to $m-1$.

Lemma 8. Given an element $\phi$ in $R_{0}$, there is a small endomorphism $\sigma$ of $B$ and an element $\psi \in R_{0}$ such that $\varphi=\psi+\sigma$ and $\psi$ is a finite Z-linear combination of the form

$$
\psi=\sum k_{i} P_{i}+\sum m_{i j} E_{i} P_{j}+\sum m_{i j}^{\prime} F_{i} P_{j} .
$$

Furthermore, if $\varphi \doteq 0$ on $B[p]$, then each of the coefficients in $\left(^{*}\right)$ is divisible by $p$.

Proof. We first observe that by restricting attention to the subgroup $B_{m}$ and applying elementary matrix theory, we obtain:

$$
\begin{array}{lll}
P_{j} E_{k}=E_{k-j} P_{j}, & & 0 \leqq k<m, 0 \leqq k-j<m . \\
P_{j} E_{k}=F_{j-k} P_{j}, & & 0 \leqq k<j<m . \\
P_{j} F_{k}=F_{k+j} P_{j}, & & 0<k<m, 0<k+j<m . \\
P_{j} F_{k}=E_{-(k+j)} P_{j}, & & 0<k \leqq-j<m .
\end{array}
$$

Furthermore, formulas (2) and (3) cover the cases in which $0 \leqq k<m / 2$, $0 \leqq|j|<m / 2$, while (4) and (5) cover the cases $1 \leqq k<m / 2,0 \leqq$ $|j|<m / 2$. Thus, by choosing $M$ sufficiently large $\varphi$ is equal to an element of the form of $\psi$ on $\sum\left\{B_{m} \mid m>M\right\}$, whence $p^{M}(\varphi-\psi)=0$ and $\varphi=\psi+\sigma$ for some $\sigma \in E_{s}(B)$. Now, if $\varphi \doteq 0$, then $\psi \doteq 0$, so that for some integer $N, \psi=0$ on $B_{m}[p]$ for every $m>N$. But observe that $E_{i} P_{j}$ is simply the matrix with 1 in the $(i, i \oplus j)$ entry and 0 elsewhere, $F_{i} P_{j}$ has 1 in the $(m-i,(m-i) \oplus j)$ entry and 0 elsewhere, while $P_{j}$ has 1 in the $(k, k \oplus j)$ entry for $k=0,1, \cdots,(m-1)$ and 0 elsewhere. Thus, by choosing $m$ sufficiently large, we conclude that if $\psi=0$ on $B_{m}[p]$, then all of the coefficients in the expression for $\psi$ must be 0 modulo $p$.

Now by Lemma 8 we can apply Theorem 1 to the ring $R_{0}$ to 
obtain a pure subgroup $G$ of $C$ containing $B$ such that

$$
E(G)=E_{s}(G)+\left(E_{s}(B)+R_{0}\right)^{-} .
$$

Then by Theorem 2 we get a pure subgroup $G^{\prime}$ of $C^{\prime}$ containing $B^{\prime}$, having $G$ as a large subgroup and such that $E\left(G^{\prime}\right)=E_{s}\left(G^{\prime}\right)+R^{\prime}$ where $R^{\prime}=\left\{\alpha \in E\left(B^{\prime}\right) \mid(B \mid \alpha) \in\left(E_{s}(B)+R_{0}\right)^{-}\right\}$.

LEMMA 9. If an endomorphism $\varphi$ of $B_{m}$ has a matrix representatation which is upper triangular, then $\varphi$ does not diminish $B^{\prime}$-height on $B_{m}$. If $\varphi$ is an endomorphism of $B_{m}$ which does not diminish $B^{\prime}$ heights, then the matrix representation of $\varphi$ on $B_{m}[p]$ is upper triangular.

Proof. According to Lemma 7, $B_{m}=\bigoplus \sum_{j=0}^{m-1}\left(c_{m j}\right)$ where $c_{m j}=$ $p^{r(j)} b_{s(j)}^{\prime}$ for strictly increasing sequences $\{r(j)\}_{0}^{m-1}$ and $\{s(j)\}_{0}^{m-1}$. Now if the matrix representation of $\varphi$ is upper triangular, we have $\left(c_{m j}\right) \varphi=$ $\sum_{i=j}^{m-1} w_{j i} c_{m i}$ and, writing an element $x$ of $B_{m}$ as $x=\sum_{j=0}^{m-1} u_{j} p^{k(j)} c_{m j}$ with $p \nmid u_{j}$, we obtain $(x) \varphi=\sum_{j=0}^{m-1}\left(\sum_{i=0}^{j} w_{i j} u_{i} p^{k(i)+r(j)}\right) b_{s(j)}^{\prime}$. It is clear that $h_{B^{\prime}}(x)$ is the minimum value of $\{k(j)+r(j\}\}_{0}^{m-1}$, so that for some $j_{0}$,

$$
k(i)+r(i) \geqq k\left(j_{0}\right)+r\left(j_{0}\right)=h_{B^{\prime}}(x) .
$$

But in view of the fact that the sequence $\{r(i)\}_{0}^{m-1}$ is strictly increasing, we then infer that for $m>j \geqq i \geqq 0, k(i)+r(j) \geqq k\left(j_{0}\right)+r\left(j_{0}\right)$, from which we conclude that $h_{B^{\prime}}((x) \varphi) \geqq k\left(j_{0}\right)+r\left(j_{0}\right)=h_{B^{\prime}}(x)$. For the partial converse suppose that $\varphi$ is a $B^{\prime}$-height preserving endomorphism of $B_{m}$ and $\left(c_{m j}\right) \varphi=\sum_{i=0}^{m-1} w_{i} c_{m i}$. Then

$$
\left(p^{r(j)} b_{s(j)}^{\prime}\right) \varphi=\sum_{0}^{j-1} w_{i} p^{r(i)} b_{s(i)}^{\prime}+\sum_{j}^{m-1} w_{i} p^{r(i)} b_{s(i)}^{\prime} .
$$

But $h_{B^{\prime}}\left(p^{r(j)} b_{s(j)}^{\prime}\right)=r(j)$, while for $i \geqq j, h_{B^{\prime}}\left(w_{i} p^{r(i)} b_{s(i)}^{\prime}\right) \geqq r(i) \geqq r(j)$, so that $h_{B^{\prime}}\left(\sum_{0}^{j-1} w_{i} p^{r(i)} b_{s(i)}^{\prime}\right) \geqq r(j)$, whence $p \mid w_{i}$ for $i=0, \cdots,(j-1)$ and

$$
\left(p^{r(j)+m-1} b_{s(j)}^{\prime}\right) \varphi=\sum_{j}^{m-1} w_{i} p^{r(i)+m-1} b_{s(i)}^{\prime},
$$

which says that the matrix representation of $\phi$ on $B_{m}[p]$ is upper triangular.

THEOREM 4. Given a prime $p \neq 2$, there is a p-group $G^{\prime}$ with large subgroup $G$ such that $E(G)=\{A(G)\}$ and $E\left(G^{\prime}\right) \neq\left\{A\left(G^{\prime}\right)\right\}$.

Proof. Inasmuch as $P_{i}, 1+E_{i}$ and $1+F_{i}$ are automorphisms of $B$, we have immediately that $R_{0} \subseteq\{A(G)\}$. However, since every element of $E(G)$ is eventually equal to an element of $R_{0}$ on $G[p]$, we 
conclude from Theorem 3 that $E(G)=\{A(G)\}$.

To show that $E\left(G^{\prime}\right) \neq\left\{A\left(G^{\prime}\right)\right\}$, let us first consider an element $\psi \in E(B)$ that preserves $B^{\prime}$-height on some $\sum\left\{B_{m}[p] \mid m>M\right\}$ and is of the form

$$
\psi=\sum k_{i} P_{i}+m_{i j} E_{i} P_{j}+\sum m_{i j}^{\prime} F_{i} P_{j} .
$$

Let $D_{i}=\sum_{0}^{i-1} E_{j}, D_{i}^{\prime}=\sum_{1}^{i} F_{j}(i=1,2, \cdots)$ and $D_{0}=D_{0}^{\prime}=0$. Observe that for $0 \leqq i<m$ the matrix representation of $\left(1-D_{i}^{\prime}\right) P_{i}$ is upper triangular, while for $-m<i<0,\left(1-D_{-i}\right) P_{i}$ is strictly lower triangular. Thus, writing

$$
\psi=\sum_{i \geqq 0} k_{i}\left(1-D_{i}^{\prime}\right) P_{i}+\sum_{i<0} k_{i}\left(1-D_{-i}\right) P_{i}+\sum n_{i j} E_{i} P_{j}+\sum n_{i j}^{\prime} F_{i} P_{j},
$$

and choosing $M^{\prime}$ sufficiently large, we have that for $m>M^{\prime}, \psi$ is upper triangular on $B_{m}[p]$, while each $E_{i} P_{j}$ and $F_{i} P_{j}$ is either upper or strictly lower triangular, so that $\psi \doteq \sum_{i \geq 0} k_{i}\left(1-D_{i}^{\prime}\right) P_{i}+s$ where $s$ is some member of the ideal $S_{0}$ in $R_{0}$ generated by $\left\{E_{i}\right\}_{0}^{\infty}$ and $\left\{F_{i}\right\}_{1}^{\infty}$. Now if $\varphi$ is another endomorphism of $B$ of the same form as $\psi$ such that $\varphi$ eventually does not diminish $B^{\prime}$-height on $B$ and $\psi \varphi \doteq 1$, then $\varphi \doteq$ $\sum_{i \geqq 0} k_{i}^{\prime}\left(1-D_{i}^{\prime}\right) P_{i}+s^{\prime}$ for some $s^{\prime} \in S_{0}$, whence

$$
\sum_{i+j>0} k_{i} k_{j}^{\prime}\left(1-D_{i}^{\prime}\right) P_{i}\left(1-D_{j}^{\prime}\right) P_{j}+s^{\prime \prime} \doteq 1-k_{0} k_{0}^{\prime} 1
$$

for some $s^{\prime \prime} \in S_{0}$. However, we see from formula (4) in the proof of Lemma 8 that $P_{i}\left(1-D_{j}^{\prime}\right)=\left(1-D_{i+j}^{\prime}+D_{i}^{\prime}\right) P_{i}$ when $0<i<i+j<m$, while $\left(1-D_{i}^{\prime}\right)\left(1-D_{i+j}^{\prime}+D_{i}^{\prime}\right)=1-D_{i+j}^{\prime}$, so that

$$
\sum_{i+j>0} k_{i} k_{\jmath}^{\prime}\left(1-D_{i+j}^{\prime}\right) P_{i+j}+s^{\prime \prime} \doteq 1-k_{0} k_{0}^{\prime} 1
$$

which, by Lemma 8 yields that $p \nmid k_{0}$ but $p \mid k_{i}$ for every $i>0$. Thus $\psi \doteq k_{0} 1+s$ where $p \nmid k_{0}$ and $s \in S_{0}$.

Now suppose that $E\left(G^{\prime}\right)=\left\{A\left(G^{\prime}\right)\right\}$. Since $\left(1-D_{1}^{\prime}\right) P_{1}$ is upper triangular on $B_{m}$ for every $m$, it has an extension to an endomorphism of $G^{\prime}$ which is a sum of automorphisms $\left\{u_{i}^{\prime}\right\}_{1}^{n}$ of $G^{\prime}$. Setting $u_{i}=\left(G \mid u_{i}^{\prime}\right)$, we have that $u_{i}$ is an automorphism of $G$ which, by Lemma 8 and what we showed above, is eventually equal to $k_{i} 1+s_{i}$ on $G[p]$, where $p \nmid k_{i}$ and $s_{i} \in S_{0}$. Thus $\left(1-D_{1}^{\prime}\right) P_{1} \doteq\left(\sum_{1}^{n} k_{i}\right) 1+\sum_{1}^{n} s_{i}$ which again contradicts Lemma 8 and we conclude that $E\left(G^{\prime}\right) \neq\left\{A\left(G^{\prime}\right)\right\}$.

\section{REFERENCES}

1. F. Castagna, Sums of automorphisms of a primary abelian group, (To appear)

2. A. L. S. Corner, On endomorphism rings of primary abelian groups, Quart. J. of Math., (2) 20 (1969), 277-296.

3. L. Fuchs, On the automorphism groups of abelian p-groups, Publ. Math. Debrecen, 7 (1960), 122-129. 
4. P. Hill, Endomorphism rings generated by units, Trans. Amer. Math. Soc., 141 (1969), 99-105.

5. G. Monk, Essentially indecomposable abelian p-groups, J. London Math. Soc.

6. R. S. Pierce, Homomorphisms of Primary Abelian Groups, Topics in Abelian Groups, Scott, Foresman, and Co., Chicago, 1963.

Received June 9, 1970. Research supported by National Science Foundation grant GP-8428.

UNIVERSITY OF WASHINGTON 



\section{PACIFIC JOURNAL OF MATHEMATICS}

\section{EDITORS}

H. SAMELSON

Stanford University

Stanford, California 94305

C. R. Hовву

University of Washington

Seattle, Washington 98105
J. DugundJI

Department of Mathematics

University of Southern California

Los Angeles, California 90007

RICHARD ARENS

University of California

Los Angeles, California 90024

\section{ASSOCIATE EDITORS}
E. F. BECKENBACH
B. H. NeumanN
F. WOLF
K. YOSHIDA

\section{SUPPORTING INSTITUTIONS}

UNIVERSITY OF BRITISH COLUMBIA

CALIFORNIA INSTITUTE OF TECHNOLOGY

UNIVERSITY OF CALIFORNIA

MONTANA STATE UNIVERSITY

UNIVERSITY OF NEVADA

NEW MEXICO STATE UNIVERSITY

OREGON STATE UNIVERSITY

UNIVERSITY OF OREGON

OSAKA UNIVERSITY
UNIVERSITY OF SOUTHERN CALIFORNIA

STANFORD UNIVERSITY

UNIVERSITY OF TOKYO

UNIVERSITY OF UTAH

WASHINGTON STATE UNIVERSITY

UNIVERSITY OF WASHINGTON

$\stackrel{*}{*} \stackrel{*}{*} \stackrel{*}{*}{ }^{*}$ AMERICAN MATHEMATICAL SOCIETY

NAVAL WEAPONS CENTER 


\section{Pacific Journal of Mathematics}

\section{Vol. 41, No. $1 \quad$ November, 1972}

Anatole Beck and Peter Warren, Weak orthogonality.................

Jonnie Bee Bednar and Howard E. Lacey, Concerning Banach spaces whose duals are abstract $L$-spaces.............................

Louis Harvey Blake, Canonical extensions of measures and the extension of regularity of conditional probabilities..........................

R. A. Brooks, Conditional expectations associated with stochastic processes ..........................................

Theodore Allen Burton and Ronald Calvin Grimmer, On the asymptotic behavior of solutions of $x^{\prime \prime}+a(t) f(x)=e(t) \ldots \ldots \ldots \ldots \ldots \ldots$

Stephen LaVern Campbell, Operator-valued inner functions analytic on the closed disc ............................................

Yuen-Kwok Chan, A constructive study of measure theory...

Alexander Munro Davie and Bernt Karsten Oksendal, Peak interpolation sets for some algebras of analytic functions ...................

H. P. Dikshit, Absolute total-effective $\left(N, p_{n}\right)(c, 1)$ method ...............

Robert E. Edwards, Edwin Hewitt and Kenneth Allen Ross, Lacunarity for

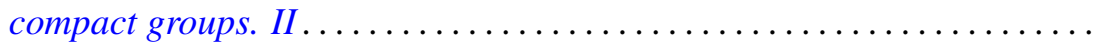

James Daniel Halpern, On a question of Tarski and a maximal theorem of Kurepa

Gerald L. Itzkowitz, A characterization of a class of uniform spaces that admit an invariant integral

Mo Tak Kiang, Semigroups with diminishing orbital diameters ..

Glenn Richard Luecke, A class of operators on Hilbert space ...

R. James Milgram, Group representations and the Adams spectral sequence. . .

G. S. Monk, On the endomorphism ring of an abelian p-group, and of a large subgroup...

Yasutoshi Nomura, Homology of a group extension ...

R. Michael Range, Approximation to bounded holomorphic functions on strictly pseudoconvex domains...

Norman R. Reilly, Inverse semigroups of partial transformations and $\theta$-classes.

Chris Rorres, Strong concentration of the spectra of self-adjoint operators

Saharon Shelah, A combinatorial problem; stability and order for models and theories in infinitary languages.

George Gustave Weill, Vector space decompositions and the abstract

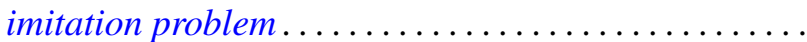

